\title{
Non-Markovian Models of Environmentally-driven Disentanglement in Molecular Charge Qubits
}

\author{
Shengyang Zhou ${ }^{1}$ and Enrique P. Blair ${ }^{1, a)}$ \\ Affiliation: Electrical and Computer Engineering Department, Baylor University, Waco, TX, USA, \\ 76798
}

(Dated: 2 March 2022)

Models of quantum disentanglement are developed for nanometer-scale molecular charge qubits (MCQs). Two MCQs, $A$ and $B$, are prepared in a Bell state and separated for negligible $A$ - $B$ interactions. Interactions between the local environment and each MCQ unravels $A$ - $B$ entanglement during coherent system+environment evolution. Three models are used for dynamics: (1) a previously-developed, numerical model, in which both $A B$ and environment $\mathcal{E}$ are modeled explicitly; (2) an exact, semi-analytic model, in which only the dynamics of $A B$ are calculated, and (3) an approximate model developed from the semi-analytic model and assumptions about randomness in $\mathcal{E}$. In the approximate model, the non-zero coherences of the density operator for $A B$ decay with a Gaussian time dependence. This provides a time scale for system dynamics in the exact models as well. This time scale is related directly to the strength of AB-E interaction. This time scale describes cases where environmental interaction with one target MCQ is dominant, generalizing a previous time scale applicable only when both MCQs have roughly the same strength of interaction with the local environment. Entanglement is measured using two-qubit correlation functions, the dynamics of which are used to demonstrate the effectiveness of the time scale. The early-time decay of coherences and the loss of entanglement is well-characterized as Gaussian, a behavior that Markovian models for memoryless environments cannot capture. The approximate Gaussian model may be used to describe the dynamics of MCQ disentanglement under the influence of environments modeled here, as well as other environments where randomness is present.

Keywords: Quantum computing, molecular charge qubit, entanglement

\section{INTRODUCTION}

Quantum computing promises new ways to process information and to efficiently solve problems that are difficult or impossible for classical computers. $\frac{122}{}$ Such applications include Shor's algorithm ${ }^{3}$ for defeating a widelyused encryption scheme, Grover's search algorithm,, 4 simulating quantum systems,, 1$]$ and optimization problems ${ }^{[-5}$ Quantum cryptography promises provably secure methods for sharing information.6 Entanglement between qubits is an essential resource in both quantum computation and communication, but it is easily unraveled by qubit-environment interactions $[8$

Several physical implementations exist for quantum bits (qubits), and still others could be invented. This paper focuses on molecular charge qubits (MCQs), which could be implemented using $\pi$-cojugated block copolymers ${ }^{9}$ or multi-metalcentered mixed-valence molecules, suitable also for a general-purpose classical computing paradigm known as quantum-dot cellular automata (QCA)!10 12 Quality factors of $\sim 10^{3}-10^{4}$ have been reported for MCQ systems, 9 making it feasible to process information using MCQs.

In this paper, the dynamics of disentanglement are studied in MCQs using computational and analytic methods. Here, a double-quantum-dot (DQD) molecule provides an MCQ. A remotely-separated target pair of

\footnotetext{
a)Electronic mail: Electronic Address: Enrique_Blair@baylor.edu
}

MCQs is prepared in a Bell state for maximal entanglement. Vast spatial separation eliminates Coulomb coupling between the target MCQs. Each MCQ in the pair is allowed to interact Coulombically with its local environment, which consists of $M$ charge-neutral DQD molecules. This is the starting point for a time evolution, over which entanglement in $A B$ is quantified using quantum correlation functions. Here, the time dependence of disentanglement is found, along with a characteristic time scale.

This work generalizes a previously-found time scale for environmentally-driven disentanglement in the target Bell pair ${ }^{13}$ Previous work was constrained to a regime in which the strength of local environmental interactions was approximately equal for each of the two target qubits. The previously-used time scale does not generalize to cases where one MCQ in the target pair suffers the dominant environmental interaction. In this paper, a more general time scale found.

A previously-developed numerical mode ${ }^{13}$ for the dynamics of disentanglement in $A B$ is reviewed, and an exact, semi-analytic model is developed in Section II. Additionally, the semi-analytic model is used with assumptions about randomness in $\mathcal{E}$ to obtain an approximate model for the dynamics of disentanglement, as well as to obtain a time scale characteristic of those dynamics. The time scale is related directly to energies of interaction between each target MCQ and its local environment and also characterizes the dynamics of the exact models. Quantum correlation functions are used to quantify entanglement in the target MCQ pair and to demonstrate 


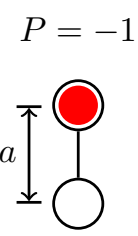

$|0\rangle$
$P=+1$

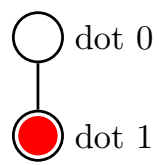

$|1\rangle$
FIG. 1. Localized electronic states of a molecular double quantum dot (DQD) system provide the two classical states of a qubit. Black circles represent the two quantum dots, and a connecting bar indicates a tunneling path. A red disc represents the mobile electron.

the effetiveness of the new time scale in characterizing the dynamics of disentanglement. The dynamics of disentanglement are seen to have a Gaussian form unattainable using Markovian models of a memoryless environment. The approximate Gaussian model for disentanglement could be used to describe $A B$ dynamics not only in the environments studied here, but also in other randomlyarranged non-Markovian environments.

\section{MODELS OF DISENTANGLEMENT}

\section{A. A Molecular Charge Qubit}

A mixed-valence compound such as diferrocenyl acetylne (DFA) can function as a molecular DQD!14|15 Here, two iron centers provide redox centers, each of which functions as a molecular quantum dot. While the DFA molecule must be singly-ionized to provide useful charge states for this application, other charge-neutral (zwitterionic) molecules are under study for both molecular charge qubits and for energy-efficient, beyond-CMOS classical computing applications. $14 \mid 1617$ In this paper, charge-neutral DQD molecules similar to DFA are considered.

Two charge-localized states of a molecular DQD provide the computational basis states for a single MCQ (See Figure 1]. Here, one mobile electron occupies one of two quantum dots. Also, a fixed charge $+e / 2$ (not pictured) is assumed to reside at each dot, providing net charge neutrality for each DQD. Here, $e$ is the fundamental charge, and the dots are treated as charged points separated by distance $a$. It will be helpful to quantify the charge state of a DQD in a single number, the polarization, $P$, given by $P=\left\langle\hat{\sigma}_{z}\right\rangle$, where $\hat{\sigma}_{z}$ is one of the Pauli operators $\left\{\hat{\sigma}_{x}, \hat{\sigma}_{y}, \hat{\sigma}_{z}\right\}$.

\section{B. A Bell Pair}

The system of interest, $A B$, is a target pair of entangled molecular charge qubits, designated $A$ and $B$. The pair $A B$ is prepared in a Bell state as the initial state of the time evolution:

$$
\left|\Psi_{A B}(0)\right\rangle=\frac{1}{\sqrt{2}}\left(\left|0_{A}\right\rangle \otimes\left|0_{B}\right\rangle+\left|1_{A}\right\rangle \otimes\left|1_{B}\right\rangle\right)
$$

Henceforth, a more compact notation is used: $\left|\Psi_{A B}(0)\right\rangle=(1 / \sqrt{2})(|00\rangle+|11\rangle)$, where $\left|m_{A} m_{B}\right\rangle=$ $\left|m_{A}\right\rangle \otimes\left|m_{B}\right\rangle$ denotes a product of $A$ and $B$ computational basis states and $m_{A}, m_{B} \in\{0,1\}$. It is assumed that $A$ and $B$ are separated spatially so that Coulomb interactions between them are negligible, but that each MCQ interacts with its own local environment. This separation could be established after preparation in $\left|\Psi_{A B}(0)\right\rangle$, or some remote entanglement mechanism could be applied after separation. The dynamics of the loss of entanglement in $A B$-not the means of entanglement - are the focus of this work.

\section{The Environment}

The local environment for each MCQ in $A B$ is explicitly modeled using $M$ DQDs surrounding each target MCQ 18 The $M$ environmental DQDs are arranged on the surface of a sphere of radius $R_{X}$ centered on qubit $X \in\{A, B\}$, as depicted in Figure 2, Here, the orientations and positions on the sphere of the environmental molecules are randomized. Generally, $R_{A} \neq R_{B}$ so that one MCQ in $A B$ may have a stronger environmental interaction than does its partner. This generalizes a previous study, in which $R_{A}=R_{B}$ was a constraint, 13 so that neither MCQ suffered the dominant environmental interaction. We designate the two local environments together as the complete environment, $\mathcal{E}$, with $N=2 M$ environmental DQDs. Environmental product states may be formed by taking tensor products

$$
\left|\vec{m}_{p}\right\rangle=\left|m_{N}\right\rangle\left|m_{N-1}\right\rangle \cdots\left|m_{k}\right\rangle \cdots\left|m_{2}\right\rangle\left|m_{1}\right\rangle
$$

where a counting number, $k$, indexes the environmental DQDs, and $m_{k} \in\{0,1\}$ labels a classical basis state for the $k$-th environmental molecule. The $N$-element binary vector,

$$
\vec{m}_{p}=m_{N} m_{N-1} \cdots m_{k} \cdots m_{2} m_{1},
$$

then, specifies an environmental product state, and $p \in$ $\left\{0,1,2, \ldots, 2^{N}-1\right\}$ is a whole-number representation of $\vec{m}_{p}$.

In this paper, the initial state of the environment, $|\mathcal{E}(0)\rangle$, is a product state of environmental DQDs, each prepared in a superposition $\left|\psi_{k}\right\rangle=$ $(1 / \sqrt{2})\left(|0\rangle+e^{i \phi_{k}}|1\rangle\right)$ :

$$
|\mathcal{E}(0)\rangle=\left|\psi_{N}\right\rangle\left|\psi_{N-1}\right\rangle \cdots\left|\psi_{2}\right\rangle\left|\psi_{1}\right\rangle .
$$

Here, the relative phase, $\phi_{k}$, is randomly selected. In the environmental classical basis, $\left\{\left|\vec{m}_{p}\right\rangle\right\}$, the state $|\mathcal{E}(0)\rangle$ 


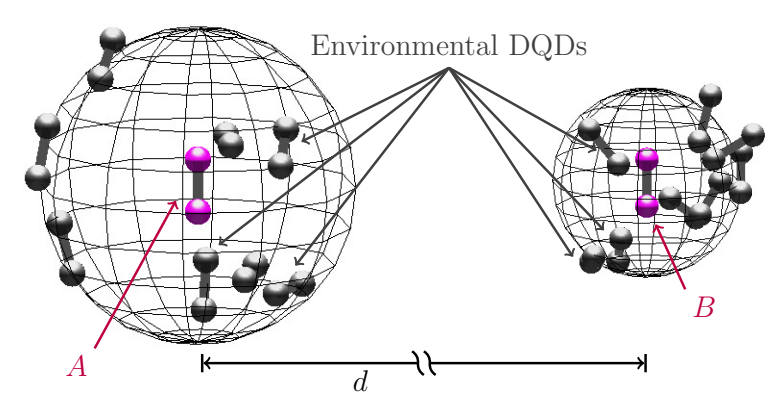

FIG. 2. The target qubits, $A$ and $B$, are entangled and coupled to local environments comprised of randomly-oriented DQDs. Colored spheres represent molecular quantum dots, and a connecting bar indicates the intramolecular tunneling path. The MCQs of the target pair are marked with purple-colored dots. There are $M$ environmental molecules distributed randomly and with random orientations about the surface of a sphere of radius $R_{X}$ for qubit $X \in\{A, B\}$. The target pair $A B$ is entangled over a large distance $d \gg R_{A}, R_{B}$ so that MCQ $A$ and its environment have negligible electrostatic interactions with MCQ $B$ and its environment.

may be written as

$$
|\mathcal{E}(0)\rangle=\frac{1}{2^{N / 2}} \sum_{\vec{m}_{p}} e^{i \Phi\left(\vec{m}_{p}\right)}\left|\vec{m}_{p}\right\rangle .
$$

where

$$
\Phi\left(\vec{m}_{p}\right)=\sum_{k=1}^{N}\left[\vec{m}_{p}\right]_{k} \phi_{k},
$$

and $\left[\vec{m}_{p}\right]_{k}$ denotes the $k$-th bit of $\vec{m}_{p}$.

\section{System Dynamics}

The Hamiltonian of the global system, $\Omega \equiv A B \mathcal{E}$, is determined by the Coulomb interactions between all the DQDs of $\Omega$. Let $U_{m_{j}, m_{k}}^{j, k}$ be the electronstatic potential energy between the $j$-th DQD in state $m_{j}$ and the $k$-th DQD in state $m_{k}$. This energy is given by

$$
U_{m_{j}, m_{k}}^{j, k}=\frac{P\left(m_{j}\right) P\left(m_{k}\right) e^{2}}{16 \pi \epsilon_{0}}\left[\frac{1}{r_{0,0}^{j, k}}-\frac{1}{r_{0,1}^{j, k}}-\frac{1}{r_{1,0}^{j, k}}+\frac{1}{r_{1,1}^{j, k}}\right],
$$

where $\epsilon_{0}$ is the permittivity of free space; $r_{m_{j}, m_{k}}^{j, k}$ is the distance between $\operatorname{dot} m_{j}$ in DQD $j$ and dot $m_{k}$ in DQD $k$; $P(m)$ is polarization of a DQD in state $m$; and $P(1)=+1$ and $P(0)=-1$.

Let $E_{m_{A} m_{B}}\left(\vec{m}_{p}\right)$ be the total electrostatic potential energy of a global state $\left|\Phi_{m_{A}, m_{B} ; \vec{m}_{p}}\right\rangle$ defined as

$$
\left|\Phi_{m_{A}, m_{B} ; \vec{m}_{p}}\right\rangle=\left|m_{A} m_{B}\right\rangle \otimes\left|\vec{m}_{p}\right\rangle .
$$

The energy $E_{m_{A} m_{B}}\left(\vec{m}_{p}\right)$ is calculated by summing over all DQD pair-wise interactions in $\Omega$ :

$$
\begin{aligned}
E_{m_{A} m_{B}}\left(\vec{m}_{p}\right) & =\left\langle\Phi_{m_{A}, m_{B} ; \vec{m}_{p}}|\hat{H}| \Phi_{m_{A}, m_{B} ; \vec{m}_{p}}\right\rangle \\
& =\frac{1}{2} \sum_{j \neq k} U_{m_{j}, m_{k}}^{j, k}
\end{aligned}
$$

Here, $\hat{H}$ is the Hamiltonian for $\Omega$, and the indices of summation, $i$ and $j$, include each DQD in $\Omega: i, j \in$ $\{A, B, 1,2, \ldots, N\}$.

To eliminate complicating dissipative effects, this study of disentanglement is constrained to the regime where tunneling between states $|0\rangle$ and $|1\rangle$ is suppressed. In this limit, the global Hamiltonian may be written as

$$
\begin{aligned}
\hat{H}= & \sum_{m_{A}, m_{B}}\left|m_{A} m_{B}\right\rangle\left\langle m_{A} m_{B}\right| \\
& \otimes \sum_{\vec{m}_{p}} E_{m_{A} m_{B}}\left(\vec{m}_{p}\right)\left|\vec{m}_{p}\right\rangle\left\langle\vec{m}_{p}\right| .
\end{aligned}
$$

The Hamiltonian is diagonal in the global basis $\left\{\left|\Phi_{m_{A} m_{B} ; \vec{m}_{p}}\right\rangle\right\}$.

\section{Global System Dynamics}

The dynamics of the global system are described exactly within this model using the Schrdinger equation,

$$
\frac{\partial}{\partial t}|\Psi(t)\rangle=-\frac{i}{\hbar} \hat{H}|\Psi(t)\rangle .
$$

The time-dependent state, $|\Psi(t)\rangle$, is obtained by applying the time evolution operator, $\hat{U}(t)=\exp (-i \hat{H} t / \hbar)$ to the initial state $|\Psi(0)\rangle$ :

$$
|\Psi(t)\rangle=\hat{U}(t)|\Psi(t)\rangle
$$

\section{Reduced Dynamics of the Target MCQ Pair}

Unlike the initial state, the time-dependent $|\Psi(t)\rangle$ generally is not a product of an $A B$ state $\left|\Psi_{A B}(t)\right\rangle$ and an environmental state $|\mathcal{E}(t)\rangle$. This is due to the interaction between $A B$ and $\mathcal{E}$, which causes entanglement between $A B$ and $\mathcal{E}$ over time, as well as the unraveling of $A-B$ entanglement.

While $A B$ may no longer have its own local state for $t>0$, the best time-dependent, local description possible for $A B$ is its reduced density matrix, $\hat{\rho}_{A B}^{(r)}(t)$. This is obtained by forming the time-dependent global density matrix, $\hat{\rho}_{\Omega}(t)=|\Psi(t)\rangle\langle\Psi(t)|$, and tracing $\hat{\rho}_{\Omega}(t)$ over the environmental degrees of freedom:

$$
\hat{\rho}_{A B}^{(r)}(t)=\operatorname{Tr}_{\mathcal{E}}\left(\hat{\rho}_{\Omega}(t)\right)=\sum_{j_{\mathcal{E}}}\left\langle j_{\mathcal{E}}\left|\hat{\rho}_{\Omega}\right| j_{\mathcal{E}}\right\rangle .
$$


Here, $\operatorname{Tr}_{\mathcal{E}}$ denotes the trace over the degrees of freedom of $\mathcal{E}$, and $\left\{\left|j_{\mathcal{E}}\right\rangle\right\}$ is any orthonormal basis for the $\mathcal{E}$. Henceforth, we drop the superscript $(r)$ from the reduced density matrix for $A B$.

This model is designated as the "numerical" model, in which the dynamics of $A B$ and $\mathcal{E}$ are calculated explicitly in order to obtain $\hat{\rho}_{A B}(t)$.

\section{E. Semi-analytic Model}

Here, an analytical treatment is used to find $\hat{\rho}_{A B}(t)$ without explicitly calculating the dynamics of $\mathcal{E}$.

The initial state vector for the system and environment is a product of the system and environment initial states from Equations (1) and (4):

$$
|\Psi(0)\rangle=\frac{1}{\sqrt{2}}(|00\rangle+|11\rangle) \otimes \frac{1}{2^{N / 2}} \sum_{\vec{m}_{p}} e^{i \Phi\left(\vec{m}_{p}\right)}\left|\vec{m}_{p}\right\rangle .
$$

Because $\hat{H}$ is diagonal in the global basis $\left\{\left|\Phi_{m_{A} m_{B} ; \vec{m}_{p}}\right\rangle\right\}$, so also is the time evolution operator, $\hat{U}(t)$ :

$$
\begin{aligned}
\hat{U}(t)= & \sum_{m_{A}, m_{B}}\left|m_{A} m_{B}\right\rangle\left\langle m_{A} m_{B}\right| \\
& \otimes \sum_{\vec{m}_{p}} e^{-i E_{m_{A} m_{B}}\left(\vec{m}_{p}\right) t / \hbar}\left|\vec{m}_{p}\right\rangle\left\langle\vec{m}_{p}\right| .
\end{aligned}
$$

Thus, the time-dependent global state $|\Psi(t)\rangle$ is found by using Equations (7), (9) and (10):

$$
\begin{aligned}
|\Psi(t)\rangle= & \frac{1}{2^{(N+1) / 2}}|00\rangle \otimes \sum_{\vec{m}_{p}} e^{-i E_{00}\left(\vec{m}_{p}\right) t / \hbar} e^{i \Phi\left(\vec{m}_{p}\right)}\left|\vec{m}_{p}\right\rangle \\
& +\frac{1}{2^{(N+1) / 2}}|11\rangle \otimes \sum_{\vec{m}_{p}} e^{-i E_{11}\left(\vec{m}_{p}\right) t / \hbar} e^{i \Phi\left(\vec{m}_{p}\right)}\left|\vec{m}_{p}\right\rangle .
\end{aligned}
$$

This may be used to form the global $\hat{\rho}_{\Omega}(t)$, which, when traced over the classical environmental basis, $\left\{\left|\vec{m}_{p}\right\rangle\right\}$, yields the reduced density matrix for the target MCQ pair:

$$
\begin{aligned}
\hat{\rho}_{A B}(t)= & \frac{1}{2}(|00\rangle\langle 00|+| 11\rangle\langle 11|) \\
& +\frac{1}{2^{N+1}}|00\rangle\langle 11| \sum_{\vec{m}_{p}} e^{-i \omega_{\mathrm{flip}}\left(\vec{m}_{p}\right) t} \\
& +\frac{1}{2^{N+1}}|11\rangle\langle 00| \sum_{\vec{m}_{p}} e^{i \omega_{\mathrm{flip}}\left(\vec{m}_{p}\right) t} .
\end{aligned}
$$

Here, we have defined the double-bit-flip frequency

$$
\omega_{\text {flip }}\left(\vec{m}_{p}\right) \equiv \frac{1}{\hbar}\left(E_{11}\left(\vec{m}_{p}\right)-E_{00}\left(\vec{m}_{p}\right)\right)=\frac{1}{\hbar} E_{\vec{m}_{p}}^{\mathrm{flip}},
$$

which is proportional to the double-bit-flip energy

$$
E_{\vec{m}_{p}}^{\mathrm{flip}}=E_{11}\left(\vec{m}_{p}\right)-E_{00}\left(\vec{m}_{p}\right)
$$

the cost of a double bit flip of $A B$ given enviromental state $\left|\vec{m}_{p}\right\rangle$.

We designate the model of Equation (11) a "semianalytic" model, since an analytic treatment was used to obtain Equation 111, but the numerous energies, $\left\{E_{\vec{m}_{p}}^{\text {flip }}\right\}$, and $\hat{\rho}_{A B}(t)$ must be evaluated numerically. This model alleviates the significant burden of explicitly calculating the dynamics of $\mathcal{E}$.

\section{F. Approximate Gaussian Model}

Now, consider the summations in Equation (11). Together with the factor $1 / 2^{N}$, these may be written as

$$
\begin{aligned}
\frac{1}{2^{N}} \sum_{\vec{m}_{p}} e^{ \pm i \omega_{\mathrm{flip}}\left(\vec{m}_{p}\right) t} & =\sum_{k} \frac{1}{k !}( \pm i t)^{k} \frac{1}{2^{N}} \sum_{\vec{m}_{p}} \omega_{\text {flip }}^{k}\left(\vec{m}_{p}\right) \\
& =\sum_{k} \frac{1}{k !}( \pm i t)^{k}\left\langle\omega^{k}\right\rangle
\end{aligned}
$$

where we define

$$
\left\langle\omega^{k}\right\rangle \equiv \frac{1}{2^{N}} \sum_{\vec{m}_{p}} \omega_{\text {flip }}^{k}\left(\vec{m}_{p}\right)
$$

Here, $\left\langle\omega^{k}\right\rangle$ is an average over $\left\{\omega_{\text {flip }}^{k}\left(\vec{m}_{p}\right)\right\}$, and we identify $\left\langle\omega^{1}\right\rangle=\bar{\omega}$ and $\sqrt{\left\langle\omega^{2}\right\rangle}=\omega_{\mathrm{RMS}}^{\mathrm{flip}}$ as average and root-meansquare values, respectively, of the frequencies $\left\{\omega_{\text {flip }}\left(\vec{m}_{p}\right)\right\}$. Similarly, we can define averages of the double-bit-flip energies and their powers:

$$
\left\langle\left(E_{\vec{m}_{p}}^{\mathrm{flip}}\right)^{k}\right\rangle \equiv \frac{1}{2^{N}} \sum_{\vec{m}_{p}}\left(E_{\vec{m}_{p}}^{\mathrm{flip}}\right)^{k}=\hbar^{n}\left\langle\omega^{k}\right\rangle
$$

with a mean double-bit-flip energy,

$$
\bar{E}_{\vec{m}_{p}}^{\mathrm{flip}}=\left\langle\left(E_{\vec{m}_{p}}^{\mathrm{flip}}\right)^{1}\right\rangle=\hbar \bar{\omega}
$$

and a root-mean-square double-bit-flip energy,

$$
\sigma_{E}=E_{\mathrm{RMS}}^{\mathrm{flip}}=\sqrt{\left\langle\left(E_{\vec{m}_{p}}^{\mathrm{flip}}\right)^{2}\right\rangle}=\hbar \omega_{\mathrm{RMS}}^{\mathrm{flip}}
$$

For environments with randomly-placed and randomly-oriented DQDs - more generally than just the spherical environments modeled in this paper-the frequencies $\left\{\omega_{\text {flip }}\left(\vec{m}_{p}\right)\right\}$ and energies $\left\{E_{\vec{m}_{p}}^{\text {flip }}\left(\vec{m}_{p}\right)\right\}$ will tend to be normally distributed. Thus, on average, a random environment will have small $\left\langle\omega^{k}\right\rangle$ and small $\left\langle\left(E_{\vec{m}_{p}}^{\mathrm{flip}}\left(\vec{m}_{p}\right)\right)^{k}\right\rangle$ for odd $k$. Neglecting these terms from Equation (14) as well as terms beyond the third order in 
$t$, we have the approximation

$$
\begin{aligned}
\frac{1}{2^{N}} \sum_{\vec{m}_{p}} e^{ \pm i \omega_{\mathrm{flip}}\left(\vec{m}_{p}\right) t}= & 1 \pm i t\left\langle\omega^{1}\right\rangle-\frac{t^{2}}{2}\left\langle\omega^{2}\right\rangle \\
& \mp i \frac{t^{3}}{3 !}\left\langle\omega^{3}\right\rangle+\cdots \\
\simeq & 1-\frac{\left(\omega_{\mathrm{RMS}}^{\mathrm{flip}} t\right)^{2}}{2} \\
\simeq & e^{-\left(\omega_{\mathrm{RMS}}^{\mathrm{flip}}\right)^{2} t^{2} / 2} .
\end{aligned}
$$

Now, inserting Equation (17) into $\hat{\rho}_{A B}(t)$ of Equation 11), the coherences $\left\langle 00\left|\hat{\rho}_{A B}\right| 11\right\rangle=\left\langle 11\left|\hat{\rho}_{A B}\right| 00\right\rangle^{*}$ have a time-dependence with a Gaussian decay:

$$
\begin{aligned}
\hat{\rho}_{A B}(t) \simeq \frac{1}{2} & {[|00\rangle\langle 00|+| 11\rangle\langle 11|} \\
& \left.+e^{-\left(\omega_{\mathrm{RMS}}^{\text {flip }}\right)^{2} t^{2} / 2}(|00\rangle\langle 11|+| 11\rangle\langle 00|)\right]
\end{aligned}
$$

The main assumption behind the Gaussian approximate model for $\hat{\rho}_{A B}(t)$ is randomness in the environment. The Gaussian model could be applied more broadly to describe the dynamics of disentanglement due to other environments where randomness is a feature, as well.

\section{Application to Local Spherical Environments}

The double-sphere environments studied in this context provide a concrete example of this analysis. Here, a histogram of the energies $\left\{E_{\vec{m}_{p}}^{f l i p}\right\}$ is plotted for a particular random environment. To provide a qualitative visual cue for how Gaussian the distribution is, a fitting function,

$$
g\left(E_{\vec{m}_{p}}^{\mathrm{flip}}\right)=A e^{-\left(E_{\vec{m}_{p}}^{\mathrm{flip}}-\bar{E}_{\vec{m}_{p}}^{\mathrm{flip}}\right)^{2} / 2 \sigma_{E}^{2}}
$$

also is plotted (dashed red curve), where $A$ is chosen to minimize curve-fitting error. The highly-Gaussian energy distribution shown in the upper panel results in a highly-Gaussian time-dependence for the magnitude of the coherences of $\hat{\rho}_{A B}$, shown in the lower panel of 3 (a). Here, the ratio $f(t)$ is plotted, which is defined as the magnitude of non-zero coherences relative to their initial magnitudes:

$$
f(t) \equiv \frac{\left|\left\langle 00\left|\hat{\rho}_{A B}(t)\right| 11\right\rangle\right|}{\left|\left\langle 00\left|\hat{\rho}_{A B}(0)\right| 11\right\rangle\right|}=\frac{\left|\left\langle 11\left|\hat{\rho}_{A B}(t)\right| 00\right\rangle\right|}{\left|\left\langle 11\left|\hat{\rho}_{A B}(0)\right| 00\right\rangle\right|} .
$$

In the plot of $f(t)$, the approximate Gaussian decay from Equation 18 is shown using a dashed red line, and deviations from this approximate behavior are attributed to the terms neglected from Equation (17).

Figure 3(b) provides an example of an environment in which the distribution $\left\{E_{\vec{m}_{p}}^{\text {flip }}\right\}$ deviates from a Gaus-

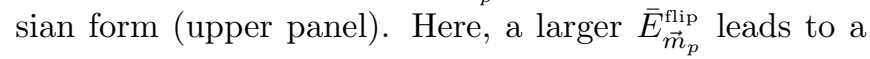

larger $\bar{\omega}$; and, other terms for higher odd powers of $t$ neglected in Equation (18) introduce non-zero imaginary components which drive departures from a purely Gaussian time dependence in the coherences of $\hat{\rho}_{A B}(t)$. Thus, more notable deviations from the red Gaussian line appear in the corresponding plot of $f(t)$ of the lower panel.

Henceforth, we refrain from calculating results using the fully numerical model, since it is more computationally-intensive than the semi-analytic treatment. This is justified, since the lower panels of subfigures 3(a) and 3(b) demonstrate exact agreement between the numerical and semi-analytic models.

\section{G. A Time Scale for Disentanglement}

Let the decay of non-zero coherences in Equation (18) be mapped to a Gaussian with standard deviation $\sigma_{t}$, $g(t) \propto \exp \left(-\left(t-t_{0}\right)^{2} / 2 \sigma_{t}^{2}\right)$. Then, for this decay, $t_{0}=$ 0 and $\sigma_{t}=1 / \omega_{\mathrm{RMS}}^{\mathrm{flip}}$. Thus, the root-mean-square doublebit-flip frequency characterizes the Gaussian decay of the coherences of $\hat{\rho}_{A B}(t)$. We define

$$
\tau_{E}=\frac{\pi}{\omega_{\mathrm{RMS}}^{\text {flip }}}=\frac{\pi \hbar}{E_{\mathrm{RMS}}^{\mathrm{flip}}} .
$$

as a time scale for the dynamics of disentanglment. Here, the factor of $\pi$ is included to make $\tau_{E}$ directly comparable to $\tau$, the time scale from previous work 13

\section{H. Measures of Entanglement}

To quantify entanglement between $A$ and $B$, we use three correlation functions: $S_{\mathrm{BM}}$, the Bell-Mermin (BM) correlation function, $\frac{19}{\sqrt{\mathrm{CHSH}}}{ }_{\mathrm{B}}$, the Clauser-HorneShimony-Holt (CHSH) correlation function, 20 and $S_{\mathrm{BPRV}}$, the Brukner-Paunković-Rudolph-Vedral (BPRV) correlation function.21 These are functions of the two-qubit reduced density matrix $\hat{\rho}_{A B}(t)$. The details of our implementations of the correlation functions are discussed either here or in the previous work by Blair, Tth, and Lent 13

\section{The Bell-Mermin Correlation Function}

The Bell-Mermin correlation used here is formulated for two qubits, $A$ and $B$, measured independently with three measurement settings, ${ }^{19} j \in\{1,2,3\}$ corresponding to three rotated angles of measurement $\left\{\theta_{j}\right\}$. The BellMermin correlation function, $S_{\mathrm{BM}}$, is

$$
S_{\mathrm{BM}}=\operatorname{Tr}\left(\hat{\rho} \hat{P}_{\text {same }}\right)
$$



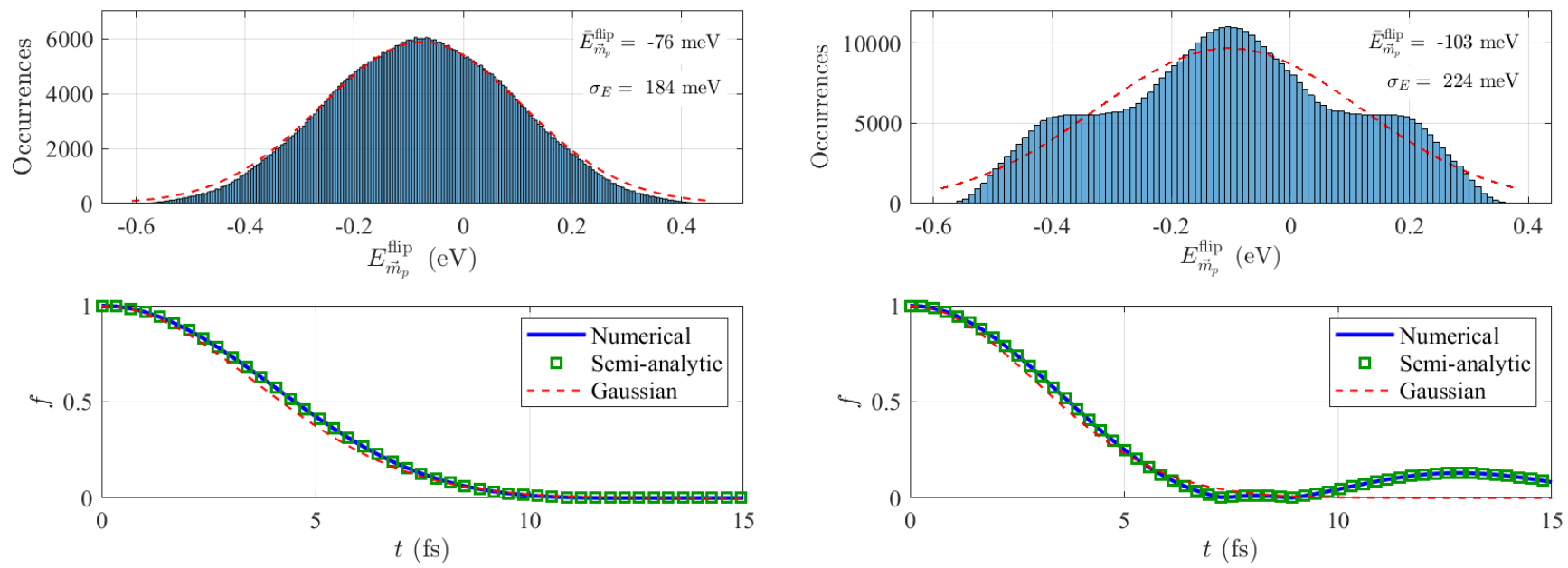

(a)

(b)

FIG. 3. As the distribution of double-bit-flip energies $\left\{E_{\vec{m}_{p}}^{\text {flip }}\right\}$ approaches a zero-centric Gaussian distribution, the time dependence of the decay in coherences $\left\langle 00\left|\hat{\rho}_{A B}\right| 11\right\rangle=\left\langle 11\left|\hat{\rho}_{A B}\right| 00\right\rangle^{*}$ becomes more Gaussian. (a) A historgram of the energies $\left\{E_{\vec{m}_{p}}^{\text {flip }}\right\}$ (upper plot) which approaches an ideal Gaussian (dashed red line) centered at the origin corresponds to a highly-Gaussian form in the decay of coherences. (b) Deviations from a zero-centric Gaussian distribution in energies $\left\{E_{\vec{m}_{p}}^{\text {flip }}\right\}$ introduces non-Gaussian behavior in the decay of $f$. In both cases shown, $a=1 \mathrm{~nm}$, the environmental radii are $R_{A} / R_{B}=4 a / 2 a$, and the environmental populations are $N=20$.

where

$$
\begin{array}{r}
\hat{P}_{\text {same }} \equiv \sum_{i=1, j \neq i}^{3} \sum_{m=0}^{1} \hat{R}\left(\theta_{i}\right)|m\rangle\langle m| \hat{R}\left(-\theta_{i}\right) \\
\otimes \hat{R}\left(\theta_{j}\right)|m\rangle\langle m| \hat{R}\left(-\theta_{j}\right),
\end{array}
$$

and $R(\theta)$ is a single-qubit rotation operator:

$$
R(\theta)=\cos \theta(|0\rangle\langle 0|+| 1\rangle\langle 1|)+\sin \theta(|0\rangle\langle 1|-| 1\rangle\langle 0|) .
$$

$S_{\mathrm{BM}}$ may be interpreted as the sum of the probabilities that a measurement on each MCQ will yield the same result, 0 or 1 , when measured in dissimilar bases. A value of $S_{\mathrm{BM}} \leq 1$ is not possible for a pair of particles described by purely classical statistics assuming local realism, so this is designated the "Bell violation" regime. To maximize the Bell violation of measurements on $\hat{\rho}_{A B}(t)$, we choose $\left(\theta_{1}, \theta_{2}, \theta_{3}\right)=(0, \pi / 3,2 \pi / 3)$.

Applying the exact, semi-analytic $\hat{\rho}_{A B}$ of Equation (11) to Equation (21), we obtain

$$
S_{\mathrm{BM}}(t)=\frac{9}{8}-\frac{3}{8} \frac{1}{2^{N}} \sum_{\vec{m}_{p}} \cos \left[\omega\left(\vec{m}_{p}\right) t\right] .
$$

The approximate $\hat{\rho}_{A B}$ of Equation (18) leads to

$$
S_{\mathrm{BM}}(t) \simeq \frac{9}{8}-\frac{3}{8} e^{-\omega_{\mathrm{RMS}}^{2} t^{2} / 2} .
$$

The approximate form of $S_{\mathrm{BM}}$ clearly highlights the initial and asymptotic values of $S_{\mathrm{BM}}(t): S_{\mathrm{BM}}(0)=3 / 4$, and
$S_{\mathrm{BM}}(\infty)=9 / 8$. Thus, the pair $A B$ starts maximallyentangled in the Bell violation regime, and time evolution unravels this entanglement through interaction and entanglement with $\mathcal{E}$.

\section{The Clauser-Horne-Shimony-Holt (CHSH) Correlation Function}

Similarly, the CHSH correlation function as implemented by Blair, Tth, and Lent ${ }^{13}$ may be applied to the semi-analytic version of $\hat{\rho}_{A B}(t)$ of Equation (11), with result

$$
S_{\mathrm{CHSH}}(t)=\sqrt{2}\left|1+\frac{1}{2^{N}} \sum_{\vec{m}_{p}} \cos \left[\omega\left(\vec{m}_{p}\right) t\right]\right| ;
$$

or the approximate $\hat{\rho}_{A B}(t)$ of Equation (18), leading to

$$
S_{\mathrm{CHSH}}(t) \simeq \sqrt{2}\left|1+e^{-\omega_{\mathrm{RMS}}^{2} t^{2} / 2}\right|
$$

Here, the Bell violation regime is $S_{\mathrm{CHSH}}>2$. By this measure of entanglement, the $A B$ pair starts well within the Bell violation region with $S_{\mathrm{CHSH}}(0)=2 \sqrt{2}$, but $A B$ eventually crosses out to a classically-describable region with $S_{\mathrm{CHSH}}(\infty)=\sqrt{2}$. 


\section{The Brukner-Paunković-Rudolph-Vedral (BPRV) Correlation Function}

Finally, the BPRV correlation is calculated for the exact $\hat{\rho}_{A B}(t)$ of Equation (11) as

$$
S_{\mathrm{BPRV}}(t)=6+\frac{3}{2^{N+1}} \sum_{\vec{m}_{p}} \cos \left[\omega\left(\vec{m}_{p}\right) t\right] .
$$

The approximate $\hat{\rho}_{A B}(t)$ of Equation results in

$$
S_{\mathrm{BPRV}}(t) \simeq 6+\frac{3}{2} e^{-\omega_{\mathrm{RMS}}^{2} t^{2} / 2} .
$$

The details of our $S_{\mathrm{BPRV}}$ calculation are found in previous work ${ }^{13}$ Here, the Bell violation regime is defined by $S_{\mathrm{BPRV}}>7$. Initially maximally entangled, $A B$ has $S_{\mathrm{BPRV}}(0)=15 / 2$, and time evolution brings $A B$ out of the Bell violation regime to an asymptotic value of $S_{\mathrm{BPRV}}(\infty)=6$.

\section{RESULTS}

\section{A. Validation of $\tau_{E}$ as a Time Scale}

Part of the motivation for this work was that $\tau=$ $\sqrt{\tau_{A} \tau_{B}}$, a previous disentanglement time scale ${ }^{13}$ used in the case where $R_{A}=R_{B}$, did not generalize well to cases in which $R_{A} \neq R_{B}$. Here, $\tau_{A}$ and $\tau_{B}$ are time scales for the decoherence of each single qubit within its own local environment.22

The limitations of $\tau$ as a time scale for disentanglement are illustrated in Figure 4. Here, the local environments are populated with $M=5$ DQDs each, and $S_{\mathrm{BM}}$ for the target MCQ pair is plotted for several randomized environments with different radial ratios, $R_{A} / R_{B}$. In particular, $R_{A}$ was fixed at $R_{A}=4 a$ and $R_{B} \in\left\{R_{A} / 2, R_{A}, 2 R_{A}\right\}$ was chosen with $a=1 \mathrm{~nm}$.

In subplot 4(a), $S_{\mathrm{BM}}$ is plotted versus time in fs for several random environments, and diverse environmental interaction strengths drive disentanglement at diverse speeds. A small $R_{B}$ results in strong $B-\mathcal{E}$ interactions (red-line cases) and drives the fastest disentanglement, as $S_{\mathrm{BM}}$ rapidly leaves the Bell violation region. On the other hand, a large $R_{B}$ generally allows the target pair to retain entanglement longer (green-line cases), up to the point where $R_{B}$ is so large that environmental interactions are dominated by $A-\mathcal{E}$ interactions, and changing $R_{B}$ no longer has a significant effect on overall $A B-\mathcal{E}$ interactions.

When each time evolution from 4(a) is time-scaled to its own particular $\tau$, as in subfigure $4(\mathrm{~b})$, the various time evolutions for the $R_{A}=R_{B}$ case roughly overlay one another, having approximately the same time-scaled form (see the blue plots). This is consistent with previous work, ${ }^{13}$ which suggests that $\tau$ is an effective time scale for characterizing disentanglement when $R_{A}=R_{B}$. On the other hand, the $\tau$-scaled calculations of $S_{\mathrm{BM}}$ with $R_{A} \neq R_{B}$ do not overlay the $\tau$-scaled $R_{A}=R_{B}$ plots, indicating that $\tau$ is not as effective a time scale when $R_{A} \neq R_{B}$. For the $R_{A} \neq R_{B}$ cases, $\tau$ overestimates the time scale for disentanglement.

Figure 5 shows $\tau_{E}$ of Equation 20 is effective at capturing the dynamics of disentanglement, even in cases where $R_{A} \neq R_{B}$. Here, several time evolutions are calculated, each for a different randomized environment. In each case, we use $a=1 \mathrm{~nm}$, and $R_{A}=4 a$, but $R_{B}$ is varied. For these time evolutions, subfigure 5(a) provides $S_{\mathrm{BM}}, S_{\mathrm{CHSH}}$, and $S_{\mathrm{BPRV}}$ plots against time in fs. As expected, a diverse range of environmental interaction strengths leads to diverse plots of the correlation functions with dynamics on different time scales. When these plots are time-scaled to $\tau_{E}$, as in subfigure 5 (b), the $\tau_{E}$-scaled correlation function plots have a common form and overlay one another for all $R_{A} / R_{B}$ ratios shown, neglecting long-time oscillations. Indeed, $\tau_{E}$ characterizes well the dynamics of disentanglement.

\section{B. Early-time Gaussian Decay of Coherences}

Figure 6 shows that the magnitudes of the coherences $\left\langle 00\left|\hat{\rho}_{A B}\right| 11\right\rangle=\left\langle 11\left|\hat{\rho}_{A B}\right| 00\right\rangle^{*}$ generally exhibit a Gaussian decay in the early-time behavior, even for $\left\{E_{\vec{m}_{p}}^{\text {flip }}\right\}$ distributions that deviate from a zero-centric Gaussian distribution and cause notable revivals in the magnitude of the coherences. To show this, a linearization technique is applied to the $f(t)$ data. A Gaussian function $g(t)=\exp \left(-t^{2} / 2 \sigma_{t}^{2}\right)$ may be linearized to obtain

$$
\ln (-\ln g)=2 \ln t-\ln \left(2 \sigma_{t}^{2}\right) .
$$

Therefore, a function $f(t)$ may be characterized as Gaussian if a plot of $y=\ln (-\ln f)$ versus $x=\ln t$ has a slope of $d y / d x=+2$. Four environments, $\left\{\mathcal{E}_{1}, \mathcal{E}_{2}, \mathcal{E}_{3}, \mathcal{E}_{4}\right\}$ were selected and characterized. Their $\left\{E_{\vec{m}_{p}}^{\text {flip }}\right\}$ distributions are shown in subfigure 6(a), and the linearization of each $f(t)$ is plotted in 6(b). For each plot, a blue line of slope +2 (labeled "Gaussian") is drawn through the leftmost data point. Since several subsequent linearized data points fall on or very close to the Gaussian marker line, we say that these time evolutions are highly Gaussian, especially at early times.

\section{DISCUSSION}

We discuss why the previously-used time scale, $\tau$, is suitable when $R_{A}=R_{B}$ but becomes less suitable when $R_{A} \neq R_{B}$.

The time scale $\tau$ was defined as the geometric mean of time scales $\tau_{A}$ and $\tau_{B}, 13$ which are time scales for decoherence of a single MCQ, $A$ or $B$, in environments $\mathcal{E}_{A}$ and $\mathcal{E}_{B}$, respectively! 22

$$
\tau=\sqrt{\tau_{A} \tau_{B}}
$$




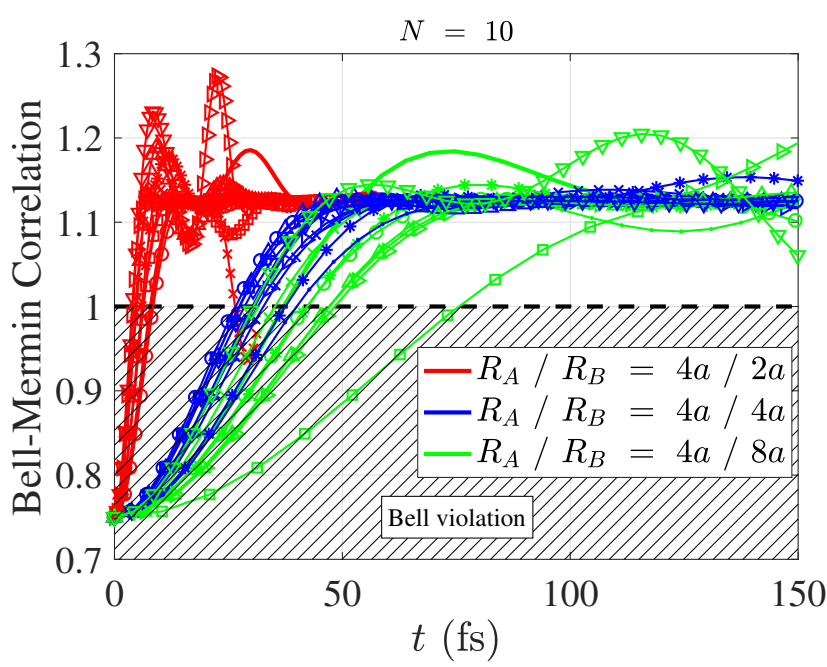

(a)

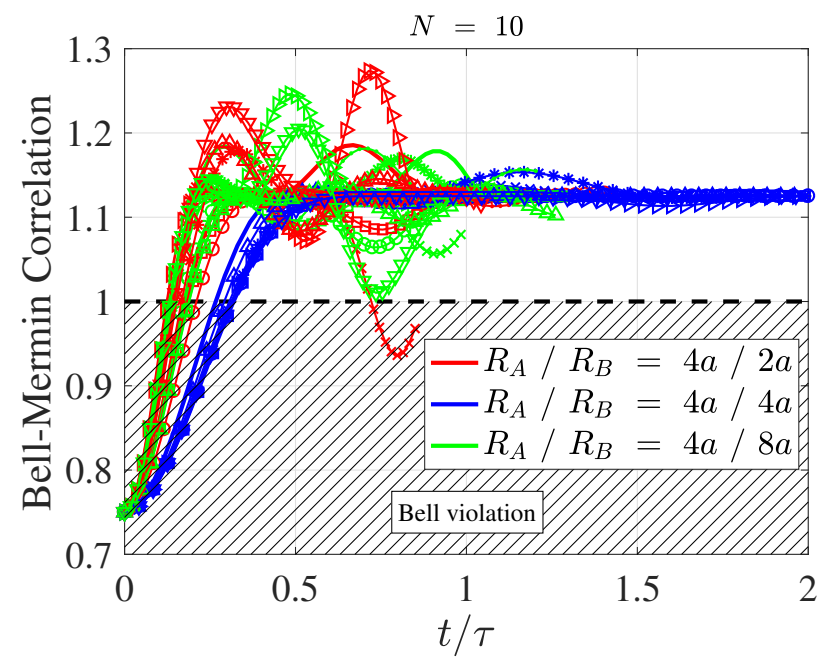

(b)

FIG. 4. A disentanglement time scale, $\tau$, characterizes the time scale of disentanglement when the two local environments interact with their individual target MCQ with roughly the same strength (that is, when $R_{A}=R_{B}$ ); however, $\tau$ does not generalize to cases where $R_{A} \neq R_{B}$. Here, $a=1 \mathrm{~nm}$, and global environmental population is $N=10$ for 3 different cases: $R_{A} / R_{B} \in\{4 a / 2 a, 4 a / 4 a, 4 a / 8 a\}$. (a) The BM correlation function, $S_{\mathrm{BM}}$, is plotted against time in fs for several time evolutions, and each randomized environment drives a unique time evolution. (b) When $S_{\mathrm{BM}}$ for each evolution is plotted against time scaled to its own $\tau, \tau$ is only partially effective as a time scale. It is most effective when $R_{A}=R_{B}$ (blue plots), mapping the various $R_{A}=R_{B}$ evolutions to roughly the same scaled time dependence. If $\tau$ also were an effective time scale for the $R_{A} \neq R_{B}$ evolutions, the red and green plots would also overlay the blue plots. However, $\tau$ overestimates the time constant when $R_{A} \neq R_{B}$.
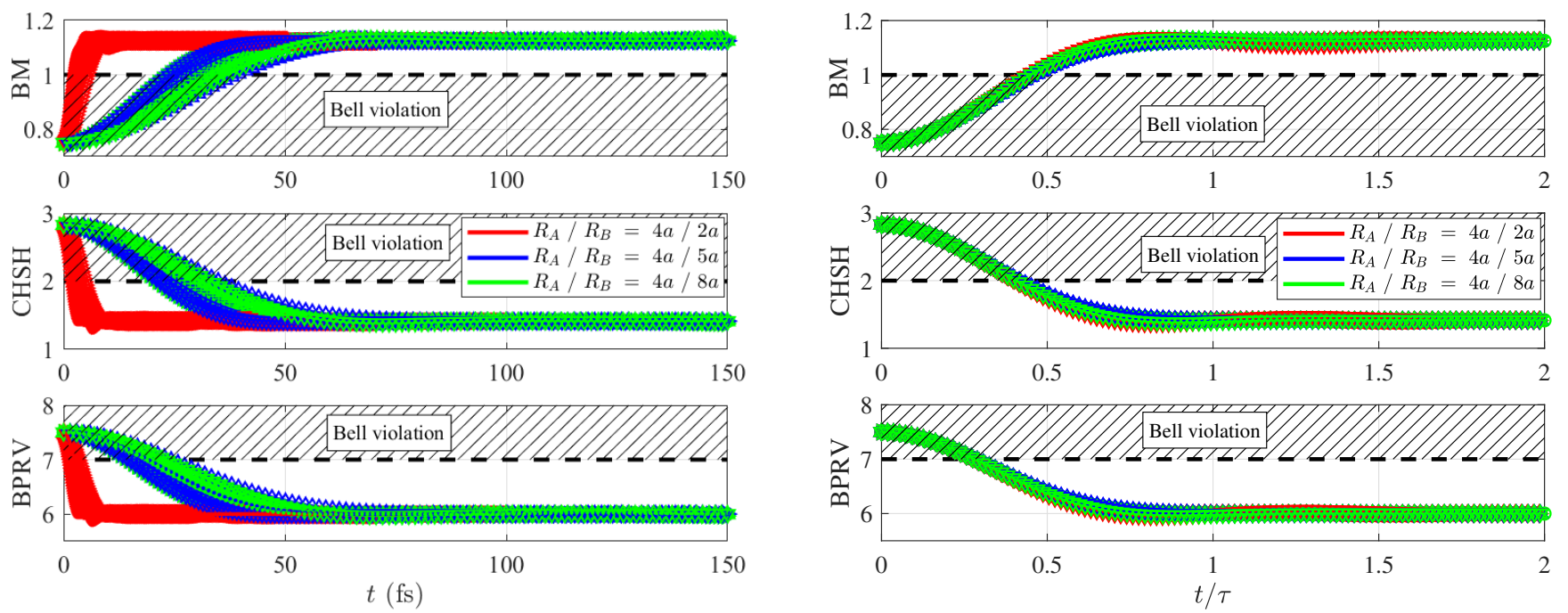

(a)

(b)

FIG. 5. The time scale $\tau_{E}$ of Equation 20 characterizes the dynamics of environmentally-driven disentangement for various ratios $R_{A} / R_{B}$. Here, $R_{A}$ is kept constant, and $R_{B}$ is varied with $a=1 \mathrm{~nm}$ and $N=20$. (a) Correlation functions from Section $[\mathrm{IIH}$ are plotted against time in fs for several random environments, showing that varied $A B-\mathcal{E}$ interaction strengths drive disentanglement over varied durations. (b) Each time evolution of subfigure (a) is time-scaled to its particular $\tau_{E}$, resulting in a common time-scaled form within each correlation function up to slight oscillations. 

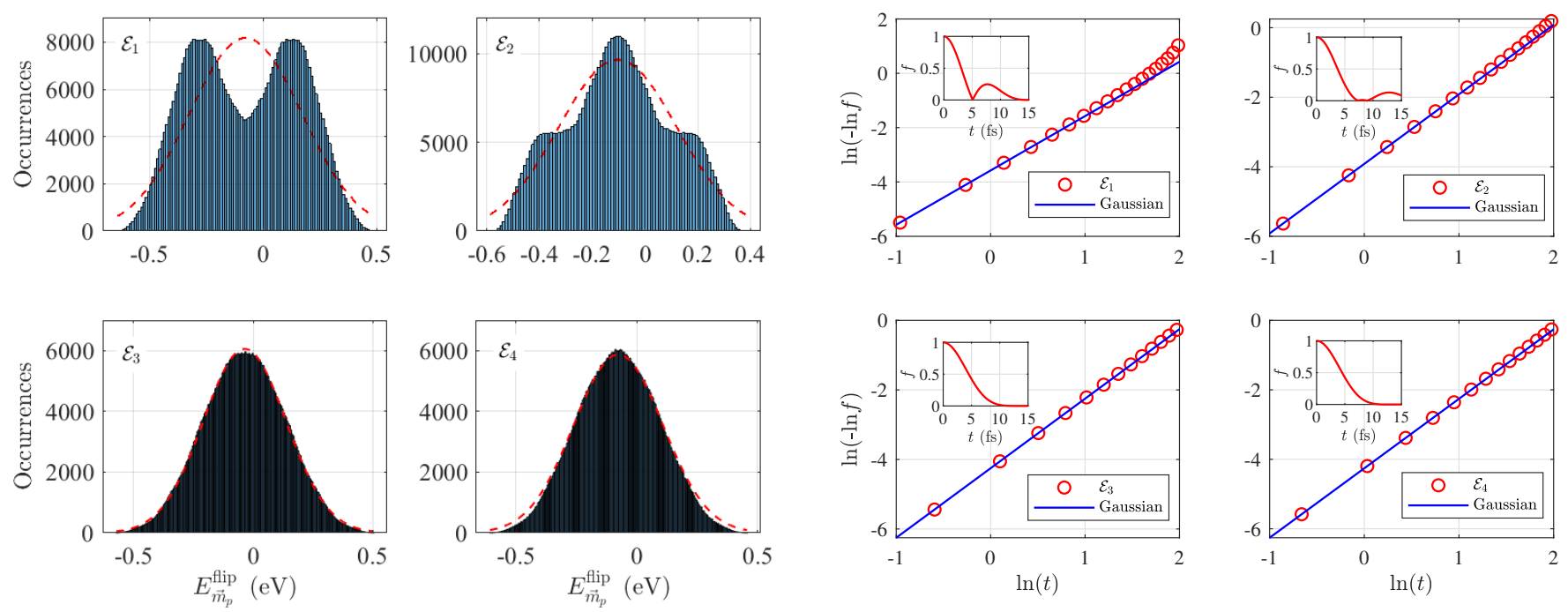

(a)
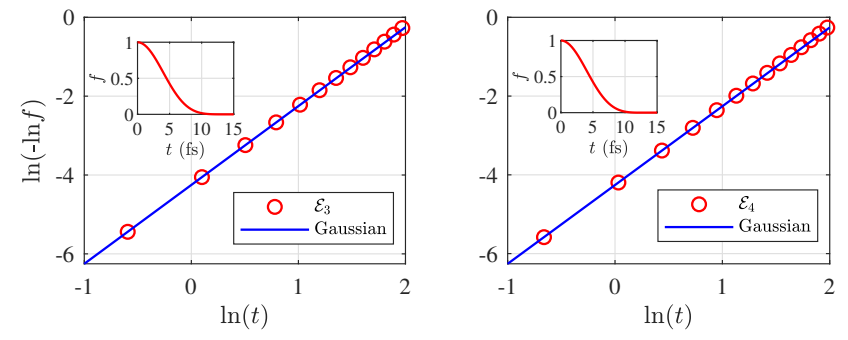

(b)

FIG. 6. Early-time behavior for both Gaussian and non-Gaussian $\left\{E_{\vec{m}_{p}}^{\mathrm{flip}}\right\}$ distributions exhibit Gaussian decay in coherences at early times. For each of the random environments with $\left\{E_{\vec{m}_{p}}^{\text {flip }}\right\}$ distributions plotted in subfigure (a), a linearization of the time-dependence of he coherences is shown in subfigure (b). Data points that exhibit Gaussian decay fall on the blue line of slope $d y / d x=2$, which marks a truly Gaussian dependence. For all environments represented in (a), early time points exhibit Gaussian decay. The time dependence of $f(t)$ is provided as an inset for each linearization panel. Here, environments with $N=20$ neighbors were used, with $a=1 \mathrm{~nm}$ and $R_{A} / R_{B}=4 a / 2 a$.

Each $\tau_{X}$ for $X \in\{A, B\}$ was defined as

$$
\tau_{X}=\frac{\pi \hbar}{E_{\mathrm{RMS}}^{(X)}},
$$

where $E_{\mathrm{RMS}}^{(X)}$ is the root-mean-square value of the singlebit-flip energies $\left\{E_{X, j}\right\}$ in evironment $\mathcal{E}_{X}$ comprised of $M$ randomly-oriented DQDs randomly placed on the surface of a shell of radius $R_{X}$ from the target MCQ:

$$
E_{\mathrm{RMS}}^{(X)}=\left(\frac{1}{2^{M}} \sum_{j=0}^{2^{M}-1}\left[E_{X}\left(\vec{m}_{X, j}\right)\right]^{2}\right)^{1 / 2} .
$$

Here, $E_{X}\left(\vec{m}_{X, j}\right)$ is the single-bit-flip energy of the target MCQ given environmental state $\left|\vec{m}_{X, j}\right\rangle$, labeled by the $M$-bit binary word

$$
\vec{m}_{X, j}=m_{M} m_{M-1} \cdots m_{2} m_{1} .
$$

Additionally, for each state $\left|\vec{m}_{X, j}\right\rangle$, there is a complementary state $\left|\vec{m}_{X, \bar{j}}\right\rangle$,

$$
\vec{m}_{X, \bar{j}}=\bar{m}_{M} \bar{m}_{M-1} \cdots \bar{m}_{k} \cdots \bar{m}_{2} \bar{m}_{1},
$$

for which the label $\vec{m}_{X, \bar{j}}$ is the bit-wise complement of $\vec{m}_{X, j}$, and for which $E_{X}\left(\vec{m}_{X, \bar{j}}\right)=-E_{X}\left(\vec{m}_{X, j}\right)$. Now, let us order $\left\{E_{X}\left(\vec{m}_{X, \bar{j}}\right)\right\}$ from most positive to most negative, and then relabel this ordered set $\left\{\varepsilon_{X, a}\right\}$, where $a$ is a counting number smaller than $2^{M}$. It is now possible to write $E_{\mathrm{RMS}}^{(X)}$ of Equation 26 in terms of only the first $2^{M-1}$ energies $\left\{\varepsilon_{X, a}\right\}$, which are non-negative by virtue of ordering:

$$
E_{\mathrm{RMS}}^{(X)}=\frac{1}{2^{(M-1) / 2}}\left(\sum_{a=0}^{2^{M-1}-1} \varepsilon_{X, a}^{2}\right)^{1 / 2}
$$

Then, $\tau=\sqrt{\tau_{A} \tau_{B}}$ is found by combining Equations 24, (25), and (27):

$$
\tau=\frac{\pi \hbar 2^{(M-1) / 2}}{\left(\left(\sum_{a=0}^{2^{M-1}-1} \varepsilon_{A, a}^{2}\right)^{1 / 2}\left(\sum_{b=0}^{2^{M-1}-1} \varepsilon_{B, b}^{2}\right)^{1 / 2}\right)^{1 / 2}} .
$$

On the other hand, there are $2^{2 M}$ double-bit-flip energies $\left\{E_{\vec{m}_{p}}^{\mathrm{flip}}\right\}$ as defined in Equation 13. These doublebit-flip energies can be formed by adding and subtracting only the positive single bit flip energies $\pm \varepsilon_{A, a}$ to $\pm \varepsilon_{B, b}$, since $A$ and $B$ do not interact: $\left\{E_{\vec{m}_{p}}^{\text {flip }}\right\}=\left\{ \pm \varepsilon_{A, a} \pm \varepsilon_{B, b}\right\}$. It can be shown that the RMS value of these double-bitflip energies is given by

$$
E_{\mathrm{RMS}}^{\mathrm{flip}}=\frac{1}{2^{(M-1) / 2}}\left(\sum_{j=0}^{2^{M-1}-1} \varepsilon_{A, j}^{2}+\varepsilon_{B, j}^{2}\right)^{1 / 2} .
$$

Thus, by combining Equations (20) and (16), the time 


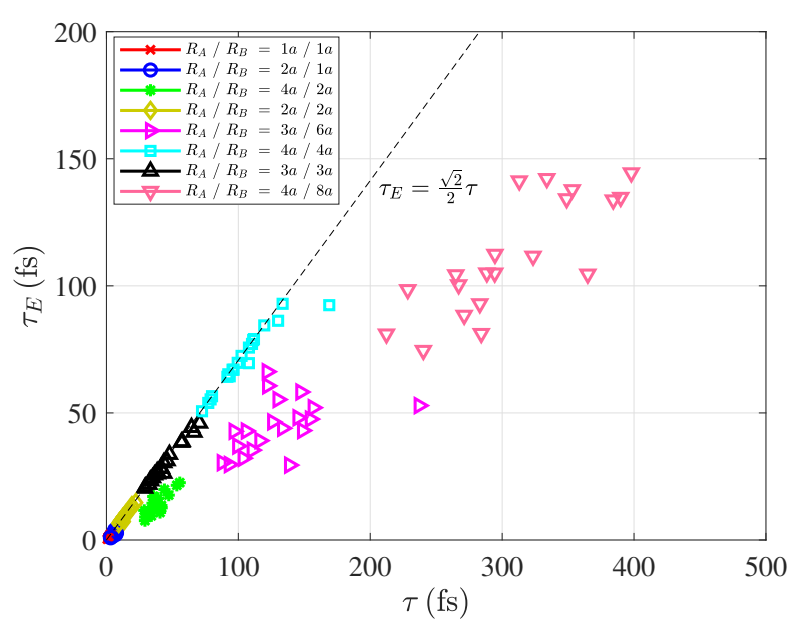

FIG. 7. When $R_{A}=R_{B}, \tau \rightarrow \tau_{E} \sqrt{2}$, and $\tau$ functions as an effective time constant for the loss of disentanglement because $\tau \propto \tau_{E}$. When $R_{A} \neq R_{B}, \tau$ deviates from proporionality to $\tau_{E}$, becoming less effective as a time constant. Here, $\tau_{E}$ and $\tau$ were calculated for several randomized environments for various $R_{A} / R_{B}$ ratios, each with $a=1 \mathrm{~nm}$ and an environmental population $N=10$.

scale $\tau_{E}$ may be written as:

$$
\tau_{E}=\frac{\pi \hbar 2^{(M-1) / 2}}{\left(\sum_{j=0}^{2^{M-1}-1} \varepsilon_{A, j}^{2}+\varepsilon_{B, j}^{2}\right)^{1 / 2}}
$$

Here, $\tau_{E}$ is written without any cross-terms, i.e. without products $\varepsilon_{A, a}^{m} \varepsilon_{B, b}^{n}$. That the total energies of interest in $\tau_{E}$ are sums of the non-negative energies $\varepsilon_{A, a}$ and $\varepsilon_{B, b}$ (and their powers) reflects the fact that $A$ and $B$ do not interact. On the other hand, cross-terms arise in the $\tau$ of Equation (28). Only when $\varepsilon_{B, j} \rightarrow \varepsilon_{A, j}$ do the cross-terms vanish from $\tau$. This is achieved approximately in our global system when $R_{A}=R_{B}$. In this case we can take the ratio of Equations (30) and 28) is $\tau_{E} / \tau \rightarrow 1 / \sqrt{2}$, and $\tau$ becomes approximately proportional to $\tau_{E}$. This proportionality between $\tau$ and $\tau_{E}$ allows $\tau$ to function as a characteristic time constant for the dynamics of disentanglement in the $R_{A}=R_{B}$ limit, as seen in previous work. 13

On the other hand, when $R_{A} \neq R_{B}$, the proportionality between $\tau_{E}$ and $\tau$ is lost, and $\tau$ fails as a characteristic time constant. This relationship is shown in the data of Figure 7. Here, a scatter plot is made for $\tau_{E}$ and $\tau$ data for various ratios of $R_{A} / R_{B}$ and several randomized environments for each ratio. When $R_{A}=R_{B}$, the points of the scatter plot fall close to the line $\tau_{E}=\tau / \sqrt{2}$; but, when $R_{A} \neq R_{B}$ the data departs from that proportionality. Mathematically, this is driven by the unphysical cross-terms arising in the approximate time constant $\tau$ when $R_{A} \neq R_{B}$.

\section{v. CONCLUSION}

In this paper, the dynamics of the loss of entanglement are studied in MCQs. Each qubit is immersed in its own local environment, modeled using a set of $M$ neighboring DQDs. Tunneling also is suppressed to eliminate dissipative effects and leave only entanglement. The systemenvironment interactions drive the the gradual loss of entanglement between $A$ and $B$. The loss of entanglement has a Gaussian from, especially at early times. This behavior is not reproducible using Markovian models of memoryless environments, which can yield only an exponential time-dependence; however, we have developed non-Markovian models, including an exact semianalytic model and an approximate Gaussian model for the density operator of the target MCQ pair. The time scale of this disentanglement is directly related to the the strength of the electrostatic interaction between the environment and the target pair of qubits. This time scale, $\tau_{E}$, describes this problem in a more general way than does a previously-developed time scale, $\tau$. While $\tau$ is useful in the case where each local environment an approximately equal strength of interaction its central target MCQ (the local environments have the same radius), $\tau_{E}$ also characterizes systems where environmental interactions are dominant for only one MCQ in the target pair (the weaker environment has a larger radius than the dominant local environment). The approximate Gaussian model may be used to provide an accurate, nonMarkovian description of system dynamics under the influence of a much broader class of environments characterized by randomness. Models of disentanglement and other quantum phenomena can help explore the dynamics of MCQs and the role they can play in quantum information processing under the influence of the environment.

\section{ACKNOWLEDGMENTS}

The authors thank Craig S. Lent from the University of Notre Dame for engaging with us in dialogue on this work. We also gratefully acknowledge peer reviewers for insightful ideas and comments, which were helpful in developing the models presented here. This work was sponsored by Baylor University under a new-faculty startup grant.

${ }^{1}$ R. Feynman, Int. J. Theor. Phys. 21, 467 (1982).

${ }^{2}$ R. Feynman, Optics news 11, 11 (1985).

${ }^{3}$ P. W. Shor, in Foundations of Computer Science, 1994 Proceedings., 35th Annual Symposium on (Ieee, 1994) pp. 124-134.

${ }^{4}$ L. Grover, unpublished (1996), arXiv:quant-ph/9605043v3.

${ }^{5}$ E. Farhi, J. Goldstone, S. Gutmann, and M. Sipser, unpublished (2000), arXiv:quant-ph/0001106v1

${ }^{6} \mathrm{C}$. Bennet and G. Brassard, in Proceedings of the IEEE International Conference on Computers, Systems and Signal Processing (1984) pp. 175-179.

${ }^{7}$ A. Ekert, Phys Rev Lett 67, 661 (1991). 
${ }^{8}$ R. Jozsa and N. Linden, Proceedings of the Royal Society of London. Series A: Mathematical, Physical and Engineering Sciences 459, 2011 (2003).

${ }^{9}$ C. Mujica-Martinez, P. Nalbach, and M. Thorwart, Phys. Rev. Lett. 111, 016802 (2013)

${ }^{10}$ C. S. Lent, Science 288, 1597 (2000).

${ }^{11}$ G. Tóth and C. Lent, Phys. Rev. A 63 (2001), 10.1103/PhysRevA.63.052315

${ }^{12}$ M. Lieberman, S. Chellamma, B. Varughese, Y. Wang, C. Lent, G. Bernstein, G. Snider, and F. Peiris, Ann. N.Y. Acad. Sci. 960, 225 (2002).

${ }^{13}$ E. P. Blair, G. Tóth, and C. S. Lent, Journal of Physics: Condensed Matter 30, 195602 (2018).

${ }^{14}$ Y. Lu and C. Lent, Chem. Phys. Lett. 582, 86 (2013)

${ }^{15}$ E. Blair, S. Corcelli, and C. Lent, J. Chem. Phys. 145, 014307 (2016).

${ }^{16}$ Y. Lu and C. Lent, Phys. Chem. Chem. Phys. 13, 14928 (2011).
${ }^{17}$ J. Christie, R. Forrest, S. Corcelli, N. Wasio, R. Quardokus, R. Brown, S. Kandel, Y. Lu, C. Lent, and K. Henderson, Angew. Chem. Int. Ed. 54, 15448 (2015).

${ }^{18}$ The MCQs and the environmental molecules all are assumed to be DQDs of the same molecular species. However, for clarity, "MCQ" is reserved for the target pair of DQDs used to model qubits; on the other hand, "DQD" is more general and may be applied to both target molecules and environmental molecules. Following this train of thought, we reserve the term "computational basis" to describe fully-localized electronic states of the MCQs in $A B$, but the term "classical basis" could describe an analogous state in any system of DQDs-either MCQ or environmental.

${ }^{19}$ N. D. Mermin, Physics today 4, 38 (1985).

${ }^{20}$ J. F. Clauser, M. A. Horne, A. Shimony, and R. A. Holt, Physical review letters 23, 880 (1969).

${ }^{21}$ Č. Brukner, N. Paunković, T. Rudolph, and V. Vedral, International Journal of Quantum Information 4, 365 (2006).

22 J. Ramsey and E. Blair, J. Appl. Phys. 122, 084304 (2017). 